\title{
Inner Product over Fuzzy Matrices
}

\author{
A. Nagoor Gani, ${ }^{1}$ K. Kannan, ${ }^{2}$ and A. R. Manikandan ${ }^{3}$ \\ ${ }^{1}$ P.G \& Research Department of Mathematics, Jamal Mohamed College (Autonomous), Tiruchirappalli 620 020, India \\ ${ }^{2}$ Department of Mathematics and Statistics, University of Jaffna, Jaffna, Sri Lanka \\ ${ }^{3}$ Department of Mathematics, Sengunthar Arts and Science College, Neikkarapatti, Tiruchengode, Tamil Nadu 637205, India
}

Correspondence should be addressed to A. Nagoor Gani; ganijmc@yahoo.co.in

Received 12 July 2015; Accepted 24 November 2015

Academic Editor: Tepper L. Gill

Copyright (C) 2016 A. Nagoor Gani et al. This is an open access article distributed under the Creative Commons Attribution License, which permits unrestricted use, distribution, and reproduction in any medium, provided the original work is properly cited.

\begin{abstract}
The purpose of this study was to introduce the inner product over fuzzy matrices. By virtue of this definition, $\alpha$-norm is defined and the parallelogram law is proved. Again the relative fuzzy norm with respect to the inner product over fuzzy matrices is defined. Moreover Cauchy Schwarz inequality, Pythagoras, and Fundamental Minimum Principle are established. Some equivalent conditions are also proved.
\end{abstract}

\section{Introduction}

The concept of fuzzy set was introduced by Zadeh [1] in 1965. Biswas [2] and El-Abyad and El-Hamouly [3] first tried to give a meaningful definition of fuzzy inner product space and associated fuzzy norm function. Also those definitions are restricted to the real linear space only. Felbin [4], Ragab, and Emam [5] introduced fuzzy 2-normed linear spaces. Meenakshi and Cokilavany [6], Bag and Samanta [7], and so forth have given different definitions of fuzzy normed linear spaces. Gani and Kalyani [8] introduced the concept of binormed sequences in fuzzy matrices. Gani and Kalyani [9] introduced the properties of fuzzy $m$-norm matrices. Gani and Kalyani Introduced the definition of fuzzy equivalence relation. Recently Majumdar and Samanta [10] and Hasankhani et al. [11] have introduced a definition of fuzzy inner product space whose associated norm is of Felbin type. Somasundaram and Beaula [12] defined the 2-fuzzy 2 -normed linear space. Some standard results in fuzzy 2normed linear spaces were extended. The organization of the paper is as follows. Section 2 provides some preliminary results which are used in this paper. In Section 3, we study some results on inner product over fuzzy matrices and the parallelogram law is proved. Section 4 is devoted to establishing Cauchy Schwarz inequality, Pythagoras, and Fundamental Minimum Principle theorem in fuzzy setting.
In this paper, we have introduced the new concept of inner product over fuzzy matrices on $M_{n}(F)$, the set of all fuzzy sets of $P^{*}\left(M_{n}(F)\right)$. We introduce the notion of $\alpha$ norm on an inner product over fuzzy matrices. With the help of it, the standard parallelogram law is proved. Some important results on inner product over fuzzy matrices are proved. Moreover Cauchy Schwarz inequality, Pythagoras, and Fundamental Minimum Principle are established. Some equivalent conditions are also proved.

\section{Preliminaries}

We consider $F=[0,1]$ the fuzzy algebra with operation $[+, \cdot]$ and the standard order " $\leq$ " where $a+b=\max \{a, b\}$ and $a \cdot b=$ $\min \{a, b\}$ for all $a, b$ in $F . F$ is a commutative semiring with additive and multiplicative identities 0 and 1 , respectively. Let $M_{M N}(F)$ denote the set of all $m \times n$ fuzzy matrices over $F$. In short, $M_{n}(F)$ is the set of all fuzzy matrices of order $n$. Define "+" and scalar multiplication in $M_{n}(F)$ as $A+B=\left[a_{i j}+b_{i j}\right]$, where $A=\left[a_{i j}\right]$ and $B=\left[b_{i j}\right]$, and $c A=\left[c a_{i j}\right]$, where $c$ is in $[0,1]$; with these operations $M_{n}(F)$ forms a linear space.

\section{3. $m$-Norm and Binorm Fuzzy Matrices}

Definition 1. Let $M_{n}(F)$ be the set of all $(n \times n)$ fuzzy matrices over $F=[0,1]$. For every $A$ in $M_{n}(F)$ define $m$-norm of $A$ 
denoted by $\|A\|_{m}$ as

$$
\begin{aligned}
\|A\|_{m} & =\max \left[a_{i j}\right], \quad \text { where } A=\left[a_{i j}\right] \text { or } \\
\|A\|_{m} & =\max \left[a_{11}, a_{12}, \ldots, a_{i j}, \ldots, a_{n n}\right] \text { or } \\
\|A\|_{m} & =\sum_{i=1}^{n} \sum_{j=1}^{n} a_{i j} .
\end{aligned}
$$

Definition 2. Let $A$ be in $M_{n}(F)$ and let $\alpha$ be in $[0,1]$ such that $\|A\|_{m}=\alpha$. Then the pair $(A, \alpha)$ is called a fuzzy point in $M_{n}(F)$ and it is denoted by $P_{A}^{\alpha}$. The dual fuzzy point for $P_{A}^{\alpha}$ is the point with $\alpha$-norm $(1-\alpha)$ denoted by $P^{*}=P^{1-\alpha}$.

Definition 3. The set of all fuzzy points in $M_{n}(F)$ is given by $P^{*}\left(M_{n}(F)\right)=\left\{P_{A}^{\alpha} / A \in M_{n}(F), \alpha \in[0,1]\right\}$

In $F$ we follow the usual $\leq$ order relation; correspondingly we define an order relation in $P^{*}\left(M_{n}(F)\right)$ as follows.

Definition 4. We define $P_{A}^{\alpha} \leq P_{B}^{\beta}$ if and only if $\alpha<\beta$ and $P_{A}^{\alpha}=P_{B}^{\beta}$ if and only if $A=B$ (then automatically $\alpha=\beta$ ).

Definition 5. A binorm with respect to the $m$-norm in $M_{n}(F)$ is a real valued function $\theta$ defined on $P^{*}\left(M_{n}(F)\right) \times P^{*}\left(M_{n}(F)\right)$ to $[0,1]$ satisfying the following conditions:

(i) $\theta\left(P_{1}, P_{2}\right)=0$ if and only if $P_{1}=P_{2}$.

(ii) $\theta\left(P_{1}, P_{2}\right)=\theta\left(P_{2}^{*}, P_{1}^{*}\right)$.

(iii) $\theta\left(\alpha P_{1}, P_{2}\right)=\alpha \theta\left(P_{1}, P_{2}\right), \forall \alpha \in[0,1]$.

(iv) $\theta\left(P_{1}+P_{2}, P_{3}\right) \leq \theta\left(P_{1}, P_{3}\right)+\theta\left(P_{2}, P_{3}\right)$.

Then $\left(M_{n}(F), \theta\right)$ is called a fuzzy binormed linear space with respect to the $m$-norm.

$\left[\theta\left(P_{1}, P_{2}\right)\right.$ is defined for $P_{1}=P_{2}$ as 0 (by (i)). Therefore $\theta\left(P_{1}, P_{2}\right) \neq 0$ is defined for $P_{1}<P_{2}$ ].

(i) When $P_{1}=P_{2}$ both the fuzzy points coincide.

Therefore $\theta\left(P_{1}, P_{2}\right)=0$.

Conversely $\theta\left(P_{1}, P_{2}\right)=0 \Rightarrow$ both fuzzy points should coincide $P_{1}=P_{2}$.

(ii) $P_{1}<P_{2} \Rightarrow \alpha_{1}<\alpha_{2} \Rightarrow 1-\alpha_{2}<1-\alpha_{1} \Rightarrow P_{2}^{*}<P_{1}^{*}$.

Therefore $\theta\left(P_{1}, P_{2}\right)=\theta\left(P_{2}^{*}, P_{1}^{*}\right)$.

(iii) Case (i): let $\alpha<\alpha_{1}$;

$$
\begin{aligned}
\theta\left(\alpha P_{1}, P_{2}\right) \Longrightarrow \alpha P_{1}<P_{2} \Longrightarrow & \alpha \alpha_{1}<\alpha_{2} \Longrightarrow \alpha<\alpha_{2} \\
& \left(\text { since } \alpha<\alpha_{1}<\alpha_{2}\right), \\
\alpha \theta\left(P_{1}, P_{2}\right) \Longrightarrow \alpha \alpha_{1}<\alpha \alpha_{2} \Longrightarrow \alpha & \alpha \Longrightarrow \alpha<\alpha_{2} \\
& \left(\text { since } \alpha<\alpha_{1}<\alpha_{2}\right) .
\end{aligned}
$$

From (2) and (3),

$$
\theta\left(\alpha P_{1}, P_{2}\right) \Longrightarrow \alpha \theta\left(P_{1}, P_{2}\right) \text {. }
$$

Case (ii): let $\alpha_{1}<\alpha$;

$$
\begin{aligned}
\theta\left(\alpha P_{1}, P_{2}\right) \Longrightarrow \alpha P_{1}<P_{2} \Longrightarrow & \alpha \alpha_{1}<\alpha_{2} \Longrightarrow \alpha<\alpha_{2} \\
& \left(\text { since } \alpha<\alpha_{1}<\alpha_{2}\right), \\
\alpha \theta\left(P_{1}, P_{2}\right) \Longrightarrow \alpha \alpha_{1}<\alpha \alpha_{2} \Longrightarrow & \alpha_{1}<\alpha_{2} \\
& \text { if } \alpha_{2}<\alpha\left(\text { since } \alpha<\alpha_{1}<\alpha_{2}\right) .
\end{aligned}
$$

From (5) and (3),

$$
\theta\left(\alpha P_{1}, P_{2}\right) \Longrightarrow \alpha \theta\left(P_{1}, P_{2}\right) .
$$

(iv) Case (i): let $\alpha_{2}<\alpha_{1}$;

$$
\begin{gathered}
P_{1}+P_{2}<P_{3} \Longrightarrow \\
\alpha_{1}+\alpha_{2}<\alpha_{3} \Longrightarrow \\
\alpha_{1}<\alpha_{3} \Longrightarrow \\
P_{1}<P_{3}, \\
\theta\left(P_{1}+P_{2}, P_{3}\right)=\theta\left(P_{1}, P_{3}\right) .
\end{gathered}
$$

Case (ii): let $\alpha_{1}<\alpha_{2}$

$$
\begin{gathered}
P_{1}+P_{2}<P_{3} \Longrightarrow \\
\alpha_{1}+\alpha_{2}<\alpha_{3} \Longrightarrow \\
\alpha_{2}<\alpha_{3} \Longrightarrow \\
P_{2}<P_{3}, \\
\theta\left(P_{1}+P_{2}, P_{3}\right)=\theta\left(P_{2}, P_{3}\right) .
\end{gathered}
$$

From (9) and (11),

$\theta\left(P_{1}+P_{2}, P_{3}\right)=$ either $\theta\left(P_{1}, P_{3}\right)$ or $\theta\left(P_{2}, P_{3}\right)$.

Therefore $\theta\left(P_{1}+P_{2}, P_{3}\right) \leq \theta\left(P_{1}, P_{3}\right)+\theta\left(P_{2}, P_{3}\right)$.

Since $\theta\left(P_{2}, P_{3}\right) \geq 0$, [in fact $\theta\left(P_{1}+P_{2}, P_{3}\right)=\theta\left(P_{1}, P_{3}\right)+$ $\left.\theta\left(P_{2}, P_{3}\right)\right]$.

Thus $\theta$ is a fuzzy binorm with respect to the $m$-norm in $M_{n}(F)$ and hence $M_{n}(F)$ is a fuzzy binormed linear space with respect to the $m$-norm.

Definition 6. Let $M_{n}(F)$ be a linear space over $K$ (the field is real or complex number). A fuzzy subset $\theta$ of $M_{n}(F) \times R$ is called a fuzzy norm of $M_{n}(F)$ if and only if, for all $P_{A}, P_{B} \in$ $P^{*}\left(M_{n}(F)\right)$ and $c$ in $K$.

$\left(\theta_{1}\right)$ For all $s \in R$ with $s \leq 0, \theta\left(P_{A}, s\right)=0$.

$\left(\theta_{2}\right)$ For all $s \in R$ with $s>0, \theta\left(P_{A}, s\right)=1$.

$\left(\theta_{3}\right)$ For all $s \in R$ with $s>0, \theta\left(c P_{A}, s\right)=\theta\left(P_{A}, s /|c|\right)$, if $c \neq 0$.

$\left(\theta_{4}\right)$ For all $s, t \in R, P_{A}, P_{B} \in P^{*}\left(M_{n}(F)\right), \theta\left(P_{A}+P_{B}, s+t\right) \geq$ $\min \left\{\theta\left(P_{A}, s\right), \theta\left(P_{B}, t\right)\right\}$. 
$\left(\theta_{5}\right) \theta\left(P_{A}, \cdot\right)$ is a nondecreasing function of $R$ and $\lim \theta\left(P_{A}\right.$, $s)=1$ as $s \rightarrow \infty$.

The pair $\left(M_{n}(F), \theta\right)$ will be referred to as a fuzzy normed linear space.

Theorem 7. Let $M_{n}(F)$ be nonempty and $P^{*}\left(M_{n}(F)\right)$ be the set of all fuzzy sets in $M_{n}(F)$. If $P^{*}\left(M_{n}(F)\right)=\left\{P_{A}^{\alpha} / A \in M_{n}(F)\right.$ and $\alpha \in[0,1]\}$ and $P_{A}$ is a bounded function for $\left|P_{A}\right| \leq 1$.

Let $K$ be the space of real numbers; then $M_{n}(F)$ is a linear space over the field $K$; where the addition and scalar multiplication are defined by

$$
\begin{aligned}
P_{A}+P_{B} & =\{(A, \alpha)+(B, \beta)\} \\
& =\left\{\frac{(A+B, \alpha \wedge \beta)}{(A, \alpha)} \in P_{A},(B, \beta) \in P_{B}\right\}, \\
k P_{B} & =\left\{\frac{\left(k P_{A}, \alpha\right)}{(A, \alpha)} \in P_{A}\right\},
\end{aligned}
$$

Proof. $M_{n}(F)$ is said to be fuzzy normed space if to every $P_{A} \in$ $P^{*}\left(M_{n}(F)\right)$ there is an associated nonnegative real number $\theta\left(P_{A}\right)$ called the $\alpha$-norm of $P_{A}$ in such a way that

(i)

$$
\begin{aligned}
\theta\left(P_{A}\right) & =0 \quad \text { iff } P_{A}=0, \\
\theta\left(P_{A}\right) & =0 \Longrightarrow \\
\left\{\frac{P_{A}^{\alpha}}{A} \in M_{n}(F), \alpha \in[0,1]\right\} & =0 \Longrightarrow \\
A & =0, \quad \alpha \text { in }[0,1] \Longrightarrow \\
P_{A} & =0 ;
\end{aligned}
$$

therefore $\theta\left(P_{A}\right)=0$ if and only if $\left[P_{A}\right]=0$;

(ii) one has

$$
\begin{aligned}
\theta\left(k P_{A}\right) & =|k| \theta\left(P_{A}\right), \quad \forall k \text { in } K, \\
\theta\left(k P_{A}\right) & =\left\{\frac{\theta(k(A, \alpha))}{(A, \alpha)} \in M_{n}(F), k \in K\right\} \\
& =\left\{\frac{|k| \theta(A, \alpha)}{A} \in M_{n}(F), \alpha \in[0,1]\right\}, \\
\theta\left(k P_{A}\right) & =|k| \theta\left(P_{A}\right) ;
\end{aligned}
$$

(iii) one has

$$
\theta\left(P_{A}+P_{B}\right) \leq \theta\left(P_{A}\right)+\theta\left(P_{B}\right)
$$

$$
\text { for every, } P_{A}, P_{B} \in P^{*}\left(M_{n}(F)\right) \text {. }
$$

For

$$
\begin{aligned}
\theta & \left(P_{A}+P_{B}\right) \\
& =\left\{\theta(A, \alpha)+\frac{\theta(B, \beta)}{A}, B \in M_{n}(F), \alpha, \beta \in[0,1]\right\} \\
& =\left\{\frac{(A+B, \alpha \wedge \beta)}{A}, B \in M_{n}(F), \alpha, \beta \in[0,1]\right\} \\
& \leq\left\{\theta(A, \alpha \wedge \beta)+\frac{\theta(B, \alpha \wedge \beta)}{(A, \alpha)},(B, \beta) \in M_{n}(F)\right\}, \\
\theta & \left(P_{A}+P_{B}\right) \leq \theta\left(P_{A}\right)+\theta\left(P_{B}\right) .
\end{aligned}
$$

Therefore $\left(M_{n}(F), \theta\right)$ is a fuzzy normed linear space.

\section{Inner Product over Fuzzy Matrices}

Definition 8. Let $M_{n}(F)$ be a linear space over the filed $C$. The fuzzy subset $\eta$ defined a mapping from $P^{*}\left(M_{n}(F)\right) \times$ $P^{*}\left(M_{n}(F)\right) \times C$ to $[0,1]$ such that, for all $P_{A}, P_{B}, P_{g} \in$ $P^{*}\left(M_{n}(F)\right), \alpha \in C$ :

(i) For $s, t \in C, \eta\left(P_{A}+P_{B}, P_{g},|s|+|t|\right) \geq \min \left\{\eta\left(P_{A}, P_{g},|s|\right)\right.$, $\left.\eta\left(P_{B}, P_{g},|t|\right)\right\}$.

(ii) For $s, t \in C, \eta\left(P_{A}+P_{B},|s t|\right) \geq \min \left\{\eta\left(P_{A}, P_{A},|s|^{2}\right)\right.$, $\left.\eta\left(P_{B}, P_{B},|t|^{2}\right)\right\}$

(iii) For $s, t \in C, \eta\left(P_{A}, P_{B}, s\right)=\eta\left(P_{B}, P_{A}, \bar{s}\right)$.

(iv) For $s \in C, \eta\left(k P_{A}, P_{B},|s|\right)=\eta\left(P_{A}, P_{B}, s /|k|\right)$, for all $\alpha(\neq 0) \in C$.

(v) $\eta\left(P_{A}, P_{A}, s\right)=0$ for all $s \in C \backslash R^{+}$.

(vi) $\eta\left(P_{A}, P_{A}, s\right)=1$ for all $s>0$ if and only if $P_{A}=0$.

(vii) $\eta\left(P_{A}, P_{A}, \cdot\right): R \rightarrow I(=[0 ; 1])$ is a monotonic nondecreasing function of $R$ and $\lim \eta\left(P_{A}, P_{A}, s\right)=1$ as $s \rightarrow$ $\infty$.

Then $\eta$ is said to be 2 -fuzzy inner product (2-FIP) on $M_{n}(F)$ and the pair $\left(M_{n}(F), \theta\right)$ is called a 2 -fuzzy inner product space (2-FIPS).

Example 9. If

$$
\begin{aligned}
& P_{A}=\left[\begin{array}{lll}
0.8 & 0.3 & 0.2 \\
0.6 & 0.9 & 0.6 \\
0.1 & 0.7 & 0.8
\end{array}\right], \\
& P_{B}=\left[\begin{array}{lll}
0.2 & 0.3 & 0.7 \\
0.7 & 0.5 & 0.6 \\
0.8 & 0.6 & 0.7
\end{array}\right],
\end{aligned}
$$

$$
P_{g}=\left[\begin{array}{lll}
0.3 & 0.6 & 0.7 \\
0.1 & 0.5 & 0.2 \\
0.2 & 0.6 & 0.3
\end{array}\right],
$$




$$
\begin{aligned}
& s=\left[\begin{array}{lll}
0.7 & 0.4 & 0.5 \\
0.4 & 0.8 & 0.2 \\
0.1 & 0.6 & 0.7
\end{array}\right] \\
& t=\left[\begin{array}{lll}
0.6 & 0.4 & 0.2 \\
0.4 & 0.5 & 0.3 \\
0.5 & 0.3 & 0.4
\end{array}\right] \\
& P_{A}=\left[\begin{array}{lll}
0.8 & 0.3 & 0.2 \\
0.6 & 0.9 & 0.6 \\
0.1 & 0.7 & 0.8
\end{array}\right], \\
& \left\|P_{A}\right\|=0.8\left[\begin{array}{ll}
0.9 & 0.6 \\
0.7 & 0.8
\end{array}\right]+0.3\left[\begin{array}{ll}
0.6 & 0.6 \\
0.1 & 0.8
\end{array}\right] \\
& +0.2\left[\begin{array}{ll}
0.6 & 0.9 \\
0.1 & 0.7
\end{array}\right] \\
& =0.8[0.8+0.6]+0.3[0.6+0.1] \\
& +0.2[0.6+0.1] \\
& =0.8+0.3+0.2 \text {, } \\
& \left\|P_{A}\right\|=0.8 \\
& P_{B}=\left[\begin{array}{lll}
0.2 & 0.3 & 0.7 \\
0.7 & 0.5 & 0.6 \\
0.8 & 0.6 & 0.7
\end{array}\right], \\
& \left\|P_{B}\right\|=0.2\left[\begin{array}{ll}
0.5 & 0.6 \\
0.6 & 0.7
\end{array}\right]+0.3\left[\begin{array}{ll}
0.7 & 0.6 \\
0.8 & 0.7
\end{array}\right] \\
& +0.7\left[\begin{array}{ll}
0.7 & 0.5 \\
0.8 & 0.6
\end{array}\right] \\
& =0.2[0.5+0.6]+0.3[0.7+0.6] \\
& +0.7[0.6+0.5] \\
& =0.2+0.3+0.6 \\
& \left\|P_{B}\right\|=0.6 \text {, } \\
& P_{g}=\left[\begin{array}{lll}
0.3 & 0.6 & 0.7 \\
0.1 & 0.5 & 0.2 \\
0.2 & 0.6 & 0.3
\end{array}\right], \\
& \left\|P_{g}\right\|=0.3\left[\begin{array}{ll}
0.5 & 0.2 \\
0.6 & 0.3
\end{array}\right]+0.6\left[\begin{array}{ll}
0.1 & 0.2 \\
0.2 & 0.3
\end{array}\right] \\
& +0.7\left[\begin{array}{ll}
0.1 & 0.5 \\
0.2 & 0.6
\end{array}\right]
\end{aligned}
$$$$
=0.3[0.3+0.2]+0.6[0.1+0.2]
$$$$
+0.7[0.1+0.2]
$$$$
=0.3+0.2+0.2 \text {, }
$$$$
\left\|P_{g}\right\|=0.3
$$$$
s=\left[\begin{array}{lll}
0.7 & 0.4 & 0.5 \\
0.4 & 0.8 & 0.2 \\
0.1 & 0.6 & 0.7
\end{array}\right],
$$$$
\|s\|=0.7
$$$$
\bar{s}=\left[\begin{array}{lll}
0.7 & 0.4 & 0.1 \\
0.4 & 0.8 & 0.6 \\
0.5 & 0.2 & 0.7
\end{array}\right]
$$$$
\|\bar{s}\|=0.7\left[\begin{array}{ll}
0.8 & 0.6 \\
0.2 & 0.7
\end{array}\right]+0.4\left[\begin{array}{ll}
0.4 & 0.6 \\
0.5 & 0.7
\end{array}\right]
$$$$
+0.1\left[\begin{array}{ll}
0.4 & 0.8 \\
0.5 & 0.2
\end{array}\right]
$$$$
=0.7[0.7+0.2]+0.4[0.4+0.5]
$$$$
+0.1[0.2+0.5]
$$$$
=0.7+0.4+0.1 \text {, }
$$$$
\|\bar{s}\|=0.7
$$

therefore $\|s\|=\|\bar{s}\|=0.7$. Consider

$$
\begin{aligned}
t & =\left[\begin{array}{lll}
0.6 & 0.4 & 0.2 \\
0.4 & 0.5 & 0.3 \\
0.5 & 0.3 & 0.4
\end{array}\right], \\
\|t\| & =0.4 \\
\bar{t} & =\left[\begin{array}{lll}
0.6 & 0.4 & 0.5 \\
0.4 & 0.5 & 0.3 \\
0.2 & 0.3 & 0.4
\end{array}\right], \\
\|\bar{t}\| & =0.6\left[\begin{array}{ll}
0.5 & 0.3 \\
0.3 & 0.4
\end{array}\right]+0.4\left[\begin{array}{ll}
0.4 & 0.3 \\
0.2 & 0.4
\end{array}\right]+0.5\left[\begin{array}{ll}
0.4 & 0.5 \\
0.2 & 0.3
\end{array}\right] \\
& =0.6[0.4+0.3]+0.4[0.4+0.2]+0.5[0.3+0.2] \\
& =0.4+0.4+0.3, \\
\|\bar{t}\| & =0.4 .
\end{aligned}
$$


Therefore $\|t\|=\|\bar{t}\|=0.4$. One has the following:

(i) For $s, t \in C$,

$$
\begin{aligned}
& \eta\left(P_{A}+P_{B}, P_{g},|s|+|t|\right) \\
& \quad \geq \min \left\{\eta\left(P_{A}, P_{g},|s|\right), \eta\left(P_{B}, P_{g},|t|\right)\right\} \Longrightarrow \\
& \eta(0.8+0.6,0.3,0.7+0.4) \\
& \quad \geq \min \{\eta(0.8,0.4,0.7), \eta(0.6,0.3,0.4)\} \Longrightarrow \\
& \eta(0.8,0.3,0.7) \\
& \quad \geq \min \{\eta(0.8,0.4,0.7), \eta(0.6,0.3,0.4)\} \Longrightarrow \\
& \eta(0.8,0.3,0.7) \geq \eta(0.6,0.3,0.4) .
\end{aligned}
$$

(ii) For $s, t \in C$,

$$
\begin{aligned}
& \eta\left(P_{A}+P_{B},|s t|\right) \geq \min \left\{\eta\left(P_{A}, P_{A},|s|^{2}\right),\right. \\
& \left.\eta\left(P_{B}, P_{B},|t|^{2}\right)\right\} \Longrightarrow \\
& \eta(0.8+0.6,(0.7)(0.4)) \\
& \quad \geq \min \{\eta((0.8)(0.8),(0.7)(0.7)), \\
& \eta((0.6)(0.6),(0.4)(0.4))\} \Longrightarrow \\
& \eta(0.8+0.6,0.4) \geq \min \{\eta(0.8,0.7), \eta(0.6,0.4)\} \Longrightarrow \\
& \eta(0.8,0.4) \geq \eta(0.6,0.4) .
\end{aligned}
$$

(iii) For $s, t \in C$,

$$
\begin{aligned}
\eta\left(P_{A}, P_{B}, s\right) & =\eta\left(P_{B}, P_{A}, \bar{s}\right) \Longrightarrow \\
\eta(0.8,0.6,0.7) & =\eta(0.6,0.8,0.7) \Longrightarrow \\
\eta(0.8,0.6,0.7) & =\eta(0.8,0.6,0.7) .
\end{aligned}
$$

(iv) For $s, t \in C$,

$$
\eta\left(k P_{A}, P_{B},|s|\right)=\eta\left(P_{A}, P_{B}, \frac{s}{|k|}\right) .
$$

For

$$
\begin{aligned}
k & =0.9 \Longrightarrow \\
\eta((0.9)(0.8), 0.6,0.7) & =\eta(0.8,0.6,(0.7)(0.9)) \Longrightarrow \\
\eta(0.8,0.6,0.7) & =\eta(0.8,0.6,0.7) .
\end{aligned}
$$

Theorem 10. If a 2-fuzzy inner product (2-FIP) on $M_{n}(F)$ is strictly convex, and if $\min \left\{\theta\left(P_{A},|s t|\right), \theta\left(P_{B},|s t|\right)\right\} \geq$ $\min \left\{\theta\left(P_{A},|s|^{2}\right), \theta\left(P_{B},|t|^{2}\right)\right\}$, then $\theta$ are 2-fuzzy inner product on $M_{n}(F)$.
Proof. (i) For $s, t \in C$ and $P_{A}, P_{B}, P_{g} \in P^{*}\left(M_{n}(F)\right)$, we have

$$
\begin{aligned}
\eta^{\prime} & \left(P_{A}+P_{B}, P_{g},|s|+|t|\right) \\
& =\eta^{\prime}\left(P_{A}+P_{B}, P_{g},|s|+|t|+0\right) \\
& =\theta\left(P_{A}+P_{B},|s|+|t|\right) \quad \forall \theta\left(P_{g}, 0\right) \\
& =\theta\left(P_{A}+P_{B},|s|+|t|\right) \\
& \geq \min \left\{\theta\left(P_{A},|s|\right), \theta\left(P_{B},|t|\right)\right\} \\
& =\min \left\{\eta^{\prime}\left(P_{A}, P_{B},|s|\right), \eta^{\prime}\left(P_{B}, P_{g},|t|\right)\right\} .
\end{aligned}
$$

Therefore $\eta^{\prime}\left(P_{A}+P_{B}, P_{g},|s|+|t|\right)=\min \left\{\eta^{\prime}\left(P_{A}, P_{B},|s|\right), \eta^{\prime}\left(P_{B}\right.\right.$, $\left.\left.P_{g},|t|\right)\right\}$.

(ii) One has

$$
\begin{aligned}
\eta^{\prime} & \left(P_{A}, P_{B},|s t|\right)=\theta\left(P_{A},|s t|\right)=\theta\left(P_{B},|s t|\right) \\
& =\min \left\{\theta\left(P_{A},|s t|\right), \theta\left(P_{B},|s t|\right)\right\} \\
& \geq \min \left\{\theta\left(P_{A},|s|^{2}\right), \theta\left(P_{B},|t|^{2}\right)\right\} \\
& =\min \left\{\eta^{\prime}\left(P_{A}, P_{A},|s|^{2}\right), \eta^{\prime}\left(P_{B}, P_{B},|t|^{2}\right)\right\} .
\end{aligned}
$$

(iii) One has

$$
\begin{aligned}
\eta^{\prime}\left(P_{A}, P_{B}, s\right) & =\theta\left(P_{A},|s|\right)=\theta\left(P_{A},|\bar{s}|\right) \\
\eta^{\prime}\left(P_{A}, P_{B}, \bar{t}\right) & =\theta\left(P_{B},|\bar{t}|\right) \\
& =\eta^{\prime}\left(P_{B}, P_{A},|\bar{t}|\right) \\
\eta^{\prime}\left(P_{A}, P_{B}, \bar{s}\right) & =\eta^{\prime}\left(P_{B}, P_{A}, \bar{s}\right) .
\end{aligned}
$$

(iv) One has

$$
\begin{aligned}
& \eta^{\prime}\left(k P_{A}, P_{B}, s\right)=\theta\left(k P_{A},|s|\right)=\theta\left(P_{A}, \frac{|s|}{|k|}\right) \quad[k \neq 0], \\
& \eta^{\prime}\left(k P_{A}, P_{B}, s\right)=\eta^{\prime}\left(P_{A}, P_{B}, \frac{s}{|k|}\right) .
\end{aligned}
$$

(v) One has

$$
\eta^{\prime}\left(P_{A}, P_{A}, s\right)=0 \quad \forall t \text { in } C .
$$

(vi) One has

$$
\begin{aligned}
\eta^{\prime}\left(P_{A}, P_{A}, s\right) & =0 \quad \forall t>0 \Longrightarrow \\
\theta\left(P_{A}, s\right) & =1 \quad \forall t>0 \Longrightarrow \\
P_{A}=0 . &
\end{aligned}
$$

(vii) Since $\eta^{\prime}\left(P_{A}, P_{A}, \cdot\right)=\theta\left(P_{A}, \cdot\right)$ and $\theta\left(P_{A}, \cdot\right)$ is a monotonic nondecreasing function of $R$ and $\lim \theta\left(P_{A}, s\right)=1$, this implies that $\mu^{\prime}$ has also the property. Thus $\eta^{\prime}$ is a 2 -fuzzy inner product on $M_{n}(F)$. 
Definition 11. Let $\left(M_{n}(F), \theta\right)$ be a 2-FIP satisfying the condition $\left\{\eta\left(P_{A}, P_{B}, s\right)>0\right.$, when $\left.t>0\right\}$ implying that $f=0$. Then, for all $\alpha$ in $[0,1]$, define $\theta\left(P_{A}^{\alpha}\right)=\inf \left\{t: \eta\left(P_{A}, P_{A}, s^{2}\right) \geq\right.$ $\alpha\}$ as a crisp norm on $M_{n}(F)$, called the $\alpha$-norm on $M_{n}(F)$ generated by $\eta$. Now using these definitions let one define fuzzy norm on $M_{n}(F)$ and verify the conditions as follows.

Theorem 12. Let $\theta$ be a 2-FIP on $M_{n}(F)$; then $\theta: M_{n}(F) \times$ $R \rightarrow[0,1]$ defined by

$$
\begin{aligned}
& \theta\left(P_{A}, s\right)=\eta\left(P_{A}, P_{A}, s^{2}\right) \quad \text { when } s \text { in } R, s>0, \\
& \theta\left(P_{A}, s\right)=0, \quad \text { when } s \text { in } R \text { and } s \leq 0
\end{aligned}
$$

is a fuzzy $\alpha$-norm on $M_{n}(F)$.

Proof. (i) $\theta\left(P_{A}, s\right)=0$ for all $s$ in $R$ and $s \leq 0$.

(ii) For all $s>0, \eta\left(P_{A}, P_{A}, s^{2}\right)=1$ if and only if $P_{A}=0$.

It follows that $\theta\left(P_{A}, s\right)=0$ if and only if $P_{A}=0$.

(iii) For all $s>0$ and $k \neq 0$,

$$
\begin{aligned}
& \theta\left(k P_{A}, s\right)=\eta\left(k P_{A}, k P_{A}, s^{2}\right), \\
& \theta\left(k P_{A}, s\right)=\eta\left(P_{A}, P_{A}, \frac{s^{2}}{|k|}\right) .
\end{aligned}
$$

(iv) One has

$$
\theta\left(P_{A}+P_{B}, s+t\right) \geq \min \left\{\theta\left(P_{A}, s\right), \theta\left(P_{B}, t\right)\right\}
$$

for every $s, t \in R^{+}$,

$$
\begin{aligned}
& P_{A}, P_{B} \in P *\left(M_{n}(F)\right) \Longrightarrow \\
& \theta\left(P_{A}+P_{B}, s+t\right)=\eta\left(P_{A}+P_{B}, P_{A}+P_{B},(s+t)^{2}\right) \\
& =\eta\left(P_{A}+P_{B}, P_{A}+P_{B}, s^{2}+s t+s t+t^{2}\right) \\
& =\eta\left(P_{A}, P_{A}, s^{2}\right)+\eta\left(P_{A}, P_{A}, s t\right)+\eta\left(P_{B}, P_{B}, t^{2}\right) \\
& \geq \eta\left(P_{A}, P_{A}, s^{2}\right)+\eta\left(P_{B}, P_{B}, s^{2}\right), \\
& \theta\left(P_{A}+P_{B}, s+t\right)=\theta\left(P_{A}, s\right)+\theta\left(P_{B}, t\right) \text {. }
\end{aligned}
$$

Theorem 13 (Cauchy Schwarz inequality). Let $\theta$ be a 2-fuzzy inner product on $M_{n}(F), \alpha \in[0,1]$, and let $\theta$ be a $\alpha$ norm generated from 2-FIP $\eta$ on $M_{n}(F)$; then $\mid \eta\left(P_{A}, P_{B}\right.$, st $) \mid \leq$ $\theta\left(P_{A}, s\right) \theta\left(P_{B}, t\right)$, for all $P_{A}, P_{B} \in P *\left(M_{n}(F)\right)$.

Proof. We can assume that $P_{B}>0 \forall r \in R^{+} \Longrightarrow$

$$
\begin{aligned}
0 \leq & \theta\left(P_{A}-r P_{B},(s+t)^{2}\right) \\
= & \eta\left(P_{A}-r P_{B}, P_{A}-r P_{B}, s^{2}-s t-s t+t^{2}\right) \\
\leq & \eta\left(P_{A}, P_{A}, s^{2}\right)+\eta\left(P_{A},-r P_{B},-s t\right) \\
& +\eta\left(-r P_{B}, P_{A},-s t\right)+\eta\left(-r P_{B},-r P_{B}, t^{2}\right)
\end{aligned}
$$

$$
\begin{aligned}
= & \eta\left(P_{A}, P_{A}, s^{2}\right)+|r| \eta\left(P_{A}, P_{B},-\frac{s t}{|-1|}\right) \\
& +|r| \eta\left(P_{B}, P_{A},-\frac{s t}{|-1|}\right)+\eta\left(r P_{B}, r P_{B}, t^{2}\right) \\
= & \eta\left(P_{A}, P_{A}, s^{2}\right)-|r| \eta\left(P_{A}, P_{B}, 2 s t\right) \\
& +|r|^{2} \eta\left(P_{B}, P_{B}, t^{2}\right) .
\end{aligned}
$$

We can find a fuzzy sequence

$$
r \longrightarrow \frac{\eta\left(P_{A}, P_{B}, s t\right)}{\eta\left(P_{B}, P_{B}, t^{2}\right)}
$$

Then, taking limit into the above,

$$
\begin{aligned}
0 \leq & \eta\left(P_{A}, P_{A}, s^{2}\right)-\frac{\left|\eta\left(P_{A}, P_{B}, 3 s t\right)\right|}{\eta\left(P_{B}, P_{B}, t^{2}\right)} \\
& +\frac{\left|\eta\left(P_{A}, P_{B}, 2 s t\right)\right|}{\eta\left(P_{B}, P_{B}, t^{2}\right)}, \\
\left|\eta\left(P_{A}, P_{B}, s t\right)\right| \leq & \eta\left(P_{A}, P_{A}, s^{2}\right) \eta\left(P_{B}, P_{B}, t^{2}\right) .
\end{aligned}
$$

Therefore $\left|\eta\left(P_{A}, P_{B}, s t\right)\right| \leq \theta\left(P_{A}, s\right) \theta\left(P_{B}, t\right)$.

Theorem 14 (parallelogram law). Let $\theta$ be a 2-fuzzy inner product on $M_{n}(F), \alpha \in[0,1]$, and let $\theta$ be a $\alpha$-norm generated from 2-FIP $\eta$ on $M_{n}(F)$; then $\theta\left(P_{A}+P_{B}\right)_{\alpha}^{2}+\theta\left(P_{A}-P_{B}\right)_{\alpha}^{2}=$ $2 \eta\left(P_{A}, P_{A}, s^{2}\right)+2 \eta\left(P_{B}, P_{B}, t^{2}\right)$.

Proof. Consider

$$
\begin{aligned}
& \theta\left(P_{A}+P_{B}\right)_{\alpha}^{2}+\theta\left(P_{A}-P_{B}\right)_{\alpha}^{2} \\
& =\inf \left\{s^{2}, s \in R^{+}, \theta\left(P_{A}+P_{B}, s\right) \geq \alpha\right\} \\
& \quad+\inf \left\{t^{2}, t \in R^{+}, \theta\left(P_{A}-P_{B}, t\right) \geq \alpha\right\},
\end{aligned}
$$

where $\theta$ is the fuzzy $\alpha$-norm induced from $\eta$.

One has the following:

$$
\begin{aligned}
& \theta\left(P_{A}+P_{B}\right)_{\alpha}^{2}+\theta\left(P_{A}-P_{B}\right)_{\alpha}^{2}=\inf \left\{s^{2}+t^{2}, s, t\right. \\
& \left.\quad \in R^{+}, \theta\left(P_{A}+P_{B}, s\right) \geq \alpha, \theta\left(P_{A}-P_{B}, t\right) \geq \alpha\right\}, \\
& \theta\left(P_{A}+P_{B}\right)_{\alpha}^{2}+\theta\left(P_{A}-P_{B}\right)_{\alpha}^{2}=\inf \left\{s^{2}+t^{2}, s, t\right. \\
& \left.\quad \in R^{+}, \theta\left(P_{A}+P_{B}, s\right) \wedge \theta\left(P_{A}-P_{B}, t\right) \geq \alpha\right\} \Longrightarrow
\end{aligned}
$$




$$
\begin{aligned}
& \theta\left(P_{A}+P_{B}\right)_{\alpha}^{2}+\theta\left(P_{A}-P_{B}\right)_{\alpha}^{2}=\theta\left(P_{A}+P_{B}, s\right) \theta\left(P_{A}\right. \\
& \left.+P_{B}, t\right)=\eta\left(P_{A}+P_{B}, P_{A}+P_{B},(s+t)^{2}\right)+\eta\left(P_{A}\right. \\
& \left.-P_{B}, P_{A}-P_{B},(s-t)^{2}\right)=\eta\left(P_{A}+P_{B}, P_{A}+P_{B}, s^{2}\right. \\
& \left.+s t+s t+t^{2}\right)+\eta\left(P_{A}-P_{B}, P_{A}-P_{B}, s^{2}-s t-s t\right. \\
& \left.+t^{2}\right)=\eta\left(P_{A}, P_{A}, s^{2}\right)+\eta\left(P_{A}, P_{B}, s t\right)+\eta\left(P_{B}, P_{A},\right. \\
& s t)+\eta\left(P_{B}, P_{B}, t^{2}\right)+\eta\left(P_{A}, P_{A}, s^{2}\right)+\eta\left(P_{A},-P_{B}\right. \text {, } \\
& -s t)+\eta\left(-P_{B}, P_{A}, s t\right)+\eta\left(-P_{B},-P_{B}, t^{2}\right)=\eta\left(P_{A}\right. \text {, } \\
& \left.P_{A}, s^{2}\right)+\eta\left(P_{A}, P_{B}, s t\right)+\eta\left(P_{B}, P_{A}, s t\right)+\eta\left(P_{B}, P_{B},\right. \\
& \left.t^{2}\right)+\eta\left(P_{A}, P_{A}, s^{2}\right)+\eta\left(P_{A}, P_{B},-\frac{s t}{|-1|}\right)+\eta\left(P_{B},\right. \\
& \left.P_{A},-\frac{s t}{|-1|}\right)+\eta\left(P_{B}, P_{B}, t^{2}\right)=\eta\left(P_{A}, P_{A}, s^{2}\right) \\
& +\eta\left(P_{A}, P_{B}, s t\right)+\eta\left(P_{B}, P_{A}, s t\right)+\eta\left(P_{B}, P_{B}, t^{2}\right) \\
& +\eta\left(P_{A}, P_{A}, s^{2}\right)-\eta\left(P_{A}, P_{B}, s t\right)-\eta\left(P_{B}, P_{A}, s t\right) \\
& +\eta\left(P_{B}, P_{B}, t^{2}\right)=\eta\left(P_{A}, P_{A}, s^{2}\right)+\eta\left(P_{B}, P_{B}, t^{2}\right) \\
& +\eta\left(P_{A}, P_{A}, s^{2}\right)+\eta\left(P_{B}, P_{B}, t^{2}\right), \\
& \theta\left(P_{A}+P_{B}\right)_{\alpha}^{2}+\theta\left(P_{A}-P_{B}\right)_{\alpha}^{2}=2 \eta\left(P_{A}, P_{A}, s^{2}\right) \\
& +2 \eta\left(P_{B}, P_{B}, t^{2}\right) \text {. }
\end{aligned}
$$

Therefore, $\theta\left(P_{A}+P_{B}\right)_{\alpha}^{2}+\theta\left(P_{A}-P_{B}\right)_{\alpha}^{2}=2 \eta\left(P_{A}, P_{A}, s^{2}\right)+$ $2 \eta\left(P_{B}, P_{B}, t^{2}\right)$.

Theorem 15 (Pythagoras). Let $\theta$ be a 2-fuzzy inner product on $M_{n}(F), \alpha \in[0,1]$, and let $\theta$ be a $\alpha$-norm generated from 2-FIP $\eta$ on $M_{n}(F)$; then $\theta\left(P_{A}+P_{B}\right)_{\alpha}^{2}=\theta\left(P_{A}\right)_{\alpha}^{2}+\theta\left(P_{B}\right)_{\alpha}^{2}$.

Proof. Consider the following:

$$
\begin{aligned}
& \theta\left(P_{A}\right)_{\alpha}=\inf \left\{s^{2}, s \in R^{+}, \theta\left(P_{A}, s\right) \geq \alpha\right\}, \\
& \theta\left(P_{B}\right)_{\alpha}=\inf \left\{t^{2}, t \in R^{+}, \theta\left(P_{B}, t\right) \geq \alpha\right\}, \\
& \theta\left(P_{A}+P_{B}\right)_{\alpha}^{2} \\
& \quad=\inf \left\{s^{2}+t^{2}, s, t \in R^{+}, \theta\left(P_{A}, s\right)+\theta\left(P_{B}, t\right) \geq \alpha\right\}, \\
& \theta\left(P_{A}+P_{B}\right)_{\alpha}^{2} \\
& \quad=\inf \left\{s^{2}+t^{2}, s, t \in R^{+}, \theta\left(P_{A}+P_{B}, s+t\right) \geq \alpha\right\},
\end{aligned}
$$

$$
\begin{aligned}
& \theta\left(P_{A}\right)_{\alpha}^{2}+\theta\left(P_{B}\right)_{\alpha}^{2} \\
& =\inf \left\{s^{2}, s \in R^{+}, \theta\left(P_{A}, s\right) \geq \alpha\right\} \\
& \quad+\inf \left\{t^{2}, t \in R^{+}, \theta\left(P_{B}, t\right) \geq \alpha\right\} \\
& =\inf \left\{s^{2}, s \in R^{+}, \eta\left(P_{A}, P_{A}, s^{2}\right) \geq \alpha\right\} \\
& \quad+\inf \left\{t^{2}, t \in R^{+}, \eta\left(P_{B}, P_{B}, t^{2}\right) \geq \alpha\right\} \\
& =\inf \left\{s^{2}+t^{2}, s, t \in R^{+}, \theta\left(P_{A}+P_{B}, s+t\right) \geq \alpha\right\} \\
& \leq \inf \left\{s^{2}+t^{2}, s, t \in R^{+}, \theta\left(P_{A}+P_{B}, s+t\right) \geq \alpha\right\}, \\
& \theta\left(P_{A}\right)_{\alpha}^{2}+\theta\left(P_{B}\right)_{\alpha}^{2} \leq \theta\left(P_{A}+P_{B}\right)_{\alpha}^{2} \quad[\text { From }(40)] .
\end{aligned}
$$

Also

$$
\begin{aligned}
\theta\left(P_{A}\right)_{\alpha}^{2}+\theta\left(P_{B}\right)_{\alpha}^{2}=\eta\left(P_{A}, P_{A}, s^{2}\right)+\eta\left(P_{B}, P_{B}, t^{2}\right) \\
=\eta\left(P_{A}, P_{A}, s^{2}\right)+\eta\left(P_{B}, P_{B}, t^{2}\right)+\eta\left(P_{A}, P_{B}, s t\right) \\
\quad+\eta\left(P_{B}, P_{A}, s t\right) \\
\geq \inf \left\{\eta\left(P_{A}+P_{B}, P_{A}+P_{B},(s+t)^{2}\right)\right\} \\
\geq \inf \left\{s^{2}+t^{2}, s, t \in R^{+}, \theta\left(P_{A}+P_{B}, s+t\right) \geq \alpha\right\}, \\
\theta\left(P_{A}\right)_{\alpha}^{2}+\theta\left(P_{B}\right)_{\alpha}^{2} \geq \theta\left(P_{A}+P_{B}\right)_{\alpha}^{2} \quad[\text { From (40)]. }
\end{aligned}
$$

From $(*)$ and $(* *)$,

$$
\theta\left(P_{A}+P_{B}\right)_{\alpha}^{2}=\theta\left(P_{A}\right)_{\alpha}^{2}+\theta\left(P_{B}\right)_{\alpha}^{2}
$$

Definition 16. A sequence $\left\{P_{A_{n}}\right\}$ in a fuzzy $\alpha$-normed linear space $\left(M_{n}(F), \theta\right)$ is called a Cauchy sequence with respect to 2-norm if $\lim \theta\left(P_{A n}, P_{B m}\right)_{\alpha}=0$ as $n, m \rightarrow \infty$.

Definition 17. A sequence $\left\{P_{A_{n}}\right\}$ in a fuzzy $\alpha$-norm linear space $\left(M_{n}(F), \theta\right)$ is called a convergent sequence with respect to 2-norm if there exists $P_{A_{n}} \in P^{*}\left(M_{n}(F)\right)$, such that $\lim \theta\left(P_{A n}, P_{B m}\right)_{\alpha}=0$.

Definition 18. A fuzzy 2-normed linear space $\left(M_{n}(F), \theta\right)$ is said to be complete if every Cauchy sequence converges.

Definition 19. A complete fuzzy 2-normed linear space $\left(M_{n}(F), \theta\right)$ is called 2-fuzzy Banach space.

Definition 20. A complex 2-fuzzy Banach space $\left(M_{n}(F), \theta\right)$ is said to be 2-fuzzy Hilbert space if its norm is induced by the 2 -fuzzy inner product.

Theorem 21 (Fundamental Minimum Principle). A nonempty closed convex fuzzy subset $c$ of a fuzzy Hilbert space $M_{n}(F)$ has a unique element of smallest $\alpha$-norm. 
Proof. If $c$ is a 2-fuzzy convex set.

It contains $\left(P_{A}+P_{B}\right) / 2$ whenever it contains $P_{A}$ and $P_{B}$; we know that

$$
c\left(\lambda P_{A}+(1-\lambda) P_{B}\right) \geq \min \left\{c\left(P_{A}\right), c\left(P_{B}\right)\right\} .
$$

And so,

$$
c\left(\frac{P_{A}+P_{B}}{2}\right) \geq \min \left\{c\left(P_{A}\right), c\left(P_{B}\right)\right\} .
$$

Let $d=\inf \left\{\theta\left(P_{A}\right)_{\alpha}: P_{A} \in C\right\}$ where $\theta\left(P_{A}\right)_{\alpha}=\inf \{s>0$ : $\left.\eta\left(P_{A}, P_{A}, s^{2}\right) \geq \alpha\right\}$.

Let $P_{A}, P_{B}$ be a closed convex fuzzy set $C$, inside a fuzzy Hilbert space, so that both $\left|P_{A}\right|$ and $\left|P_{B}\right|$ are within $\varepsilon>0$ of the $\inf d$ of the $\alpha$-norm in $C$. Then there exists a sequence $\left\{P_{A_{n}}\right\}$ in $C$ such that $\theta\left(P_{A n}\right)_{\alpha} \rightarrow d+\varepsilon$.

By parallelogram law,

$$
\begin{gathered}
\theta\left(P_{A}+P_{B}\right)_{\alpha}^{2}+\theta\left(P_{A}-P_{B}\right)_{\alpha}^{2} \\
=2\left(\theta\left(P_{A}\right)_{\alpha}^{2}+\theta\left(P_{B}\right)_{\alpha}^{2}\right) .
\end{gathered}
$$

Since $c$ is convex,

$$
\begin{aligned}
& c\left(\frac{P_{A n}+P_{B m}}{2}\right) \geq \min \left\{c\left(P_{A n}\right), c\left(P_{B m}\right)\right\} \\
& \text { so } \frac{P_{A n}+P_{B m}}{2} \in C \\
& \theta\left(\frac{P_{A n}+P_{B m}}{2}\right) \geq d+\varepsilon \Longrightarrow \\
& \theta\left(P_{A n}+P_{B m}\right) \geq 2(d+\varepsilon), \\
& \theta\left(P_{A}-P_{B}\right)_{\alpha}^{2}=2\left(\theta\left(P_{A}\right)_{\alpha}^{2}+\theta\left(P_{B}\right)_{\alpha}^{2}\right) \\
&-\theta\left(P_{A}+P_{B}\right)_{\alpha}^{2}[\text { from }(46)] \\
& \leq 2\left(\theta\left(P_{A}\right)_{\alpha}^{2}+\theta\left(P_{B}\right)_{\alpha}^{2}\right)-4(d+\varepsilon)^{2} \\
&=2\left((d+\varepsilon)^{2}+(d+\varepsilon)^{2}\right) \\
&-4(d+\varepsilon)^{2}, \\
& \theta\left(P_{A}-P_{B}\right)_{\alpha}^{2}= 4(d+\varepsilon)^{2}-4(d+\varepsilon)^{2}=0 .
\end{aligned}
$$

$\left\{P_{A n}\right\}$ is a 2 -fuzzy Cauchy sequence in $C$, since $M_{n}(F)$ is complete and $c$ is closed convex. Thus $c$ such that $P_{A_{n}} \rightarrow P_{A}$; also $\theta\left(P_{A n}\right)_{\alpha}=\theta\left(\lim P_{A n}\right)=\lim \theta\left(P_{A n}\right)_{\alpha}=d$, which implies that $P_{A} \in C$ with smallest $\alpha$-norm. Now let us prove the uniqueness part. Suppose that there exists a $P_{B} \in C$ other than $P_{A}$ with the same $\alpha$-norm $d$.

Again, by parallelogram law,

$$
\begin{aligned}
\theta\left(\frac{P_{A n}+P_{B m}}{2}\right)_{\alpha}^{2}= & \frac{\theta\left(P_{A}\right)_{\alpha}^{2}}{2}+\frac{\theta\left(P_{B}\right)_{\alpha}^{2}}{2} \\
& -\frac{\theta\left(P_{A}-P_{B}\right)_{\alpha}^{2}}{2} \\
\leq & \frac{(d+\varepsilon)^{2}}{2}+\frac{(d+\varepsilon)^{2}}{2} \leq(d+\varepsilon)^{2} .
\end{aligned}
$$

Which is a contradiction to the definition of $d+\varepsilon$ :

$$
\theta\left(P_{A n}+P_{B m}\right) \geq 2(d+\varepsilon) .
$$

Hence there exists a unique element $P_{A} \in P^{*}\left(M_{n}(F)\right)$ of smallest $\alpha$-norm.

Theorem 22. The 2-FIP $P_{A}, P_{B} \in P^{*}\left(M_{n}(F)\right)$ is a continuous function on $P^{*}\left(M_{n_{1}}(F)\right) \times P^{*}\left(M_{n_{2}}(F)\right)$.

Proof. Let $M_{n}(F)$ be a 2-FIP and let $\left\{\left[P_{A n}\right]\right\}$ and $\left\{\left[P_{B n}\right]\right\}$ be sequence in $P^{*}\left(M_{n}(F)\right)$ such that $\theta\left(P_{A_{n}}\right)_{\alpha}=\theta\left(P_{A}\right)_{\alpha}$ and $\lim \theta\left(P_{B_{n}}\right)_{\alpha}=\theta\left(P_{B}\right)_{\alpha}$; then,

$$
\begin{aligned}
\theta & \left(\left(\left[P_{A n}\right],\left[P_{B n}\right]\right)-\left(\left[P_{A}\right],\left[P_{B}\right]\right)\right)=\theta\left(\left(\left[P_{A n}\right],\left[P_{B n}\right]\right)\right. \\
& \left.-\left(\left[P_{A n}\right],\left[P_{B}\right]\right)+\left(\left[P_{A n}\right]\left[P_{B}\right]\right)-\left(\left[P_{A}\right],\left[P_{B}\right]\right)\right) \\
& \leq \theta\left(\left(\left[P_{A n}\right],\left[P_{B n}\right]\right)-\left(\left[P_{A n}\right],\left[P_{B}\right]\right)\right) \\
& +\theta\left(\left(\left[P_{A n}\right]\left[P_{B}\right]\right)-\left(\left[P_{A}\right],\left[P_{B}\right]\right)\right) \\
& =\theta\left(\left(\left[P_{A n}\right]\left(\left[P_{B n}\right]-\left[P_{B}\right]\right)\right)\right) \\
& +\theta\left(\left(\left[P_{A n}\right]-\left[P_{A}\right]\right),\left[P_{B}\right]\right) \leq \theta\left(\left[P_{A n}\right]\right) \theta\left(\left[P_{B n}\right]\right. \\
& \left.-\left[P_{B}\right]\right)+\theta\left(\left[P_{A n}\right]-\left[P_{A}\right]\right) \theta\left(\left[P_{B}\right]\right) .
\end{aligned}
$$

Since $\left\{\left[P_{A n}\right]\right\}$ and $\left\{\left[P_{B n}\right]\right\}$ converge,

$$
\begin{aligned}
& \lim \theta\left(P_{A n}-P_{A}\right)_{\alpha}=0, \\
& \lim \theta\left(P_{B n}-P_{B}\right)_{\alpha}=0 .
\end{aligned}
$$

Theorem 23. Let $M_{n}(F)$ be a 2-FIP and $\theta$ is indeed a $\alpha$-norm on $P^{*}\left(M_{n}(F)\right)$. It also satisfies

(i) $\theta\left(P_{A}\right)>0$ for every nonzero $P_{A} \in P^{*}\left(M_{n}(F)\right)$,

(ii) $\theta\left(\left(P_{x}, P_{y}\right), P_{A}\right) \leq \theta\left(P_{x}, P_{y}\right) \theta\left(P_{A}\right)$, for all $P_{A} \in$ $P^{*}\left(M_{n}(F)\right)$ and $P_{x}, P_{y} \in P^{*}\left(M_{n}(F)\right)$.

Proof. Consider

$$
\begin{aligned}
\theta\left(t P_{A}\right)^{2} & =\theta\left(t P_{A}, t P_{A}\right) \\
& =|t| \theta\left(P_{A}, t P_{A}\right) \\
& \leq|t| \theta\left(P_{A}\right) \theta\left(t P_{A}\right), \\
\theta\left(t P_{A}\right) & \leq|t| \theta\left(P_{A}\right) .
\end{aligned}
$$

For $\lambda>0$,

$$
\begin{aligned}
& \theta\left(P_{A}\right)=\theta\left(\frac{1}{t} t P_{A}\right) \leq \frac{1}{|t|} \theta\left(t P_{A}\right), \\
& \theta\left(t P_{A}\right) \leq|t| \theta\left(P_{A}\right) \quad \forall P_{A} \in P^{*}\left(M_{n}(F)\right), t \text { in } R^{+} .
\end{aligned}
$$


Also,

$$
\begin{aligned}
\theta\left(P_{A}+P_{B}\right)_{\alpha}^{2}= & \theta\left(\left(P_{A}+P_{B}\right),\left(P_{A}+P_{B}\right)\right) \\
\leq & \theta\left(P_{A}\left(P_{A}+P_{B}\right)\right)+\theta\left(P_{B}\left(P_{A}+P_{B}\right)\right) \\
\leq & \theta\left(P_{A}\right) \theta\left(P_{A}+P_{B}\right) \\
& +\theta\left(P_{B}\right) \theta\left(P_{A}+P_{B}\right) \\
\leq & \left(\theta\left(P_{A}\right)+\theta\left(P_{B}\right)\right) \theta\left(P_{A}+P_{B}\right), \\
\theta\left(P_{A}+P_{B}\right) \leq & \theta\left(P_{A}\right)+\theta\left(P_{B}\right) .
\end{aligned}
$$

(i) Let $P_{A} \in P^{*}\left(M_{n}(F)\right)$ be a nonzero element in $M_{n}(F)$; then $P_{A} \cdot P_{A}>0$ and hence $\theta\left(P_{A}\right)=\theta\left(P_{A} \cdot P_{A}\right)^{1 / 2}>0$ in $P^{*}\left(M_{n}(F)\right)$.

(ii) Let $P_{x}, P_{y} \in P^{*}\left(M_{n}(F)\right)$;

$$
\begin{aligned}
\theta\left(\left(P_{x}, P_{y}\right), P_{A}\right)^{2} & =\theta\left(P_{x}, P_{y}\right) P_{A}, \theta\left(P_{x}, P_{y}\right) P_{A} \\
& =\theta\left(\left(P_{x}, P_{y}\right)^{2}\left(P_{A}, P_{A}\right)\right) .
\end{aligned}
$$

Since $\left(M_{n}(F), \theta\right)$ is a fuzzy norm,

$$
\begin{aligned}
& \theta\left(\left(P_{x}, P_{y}\right)^{2}\left(P_{A}, P_{A}\right)\right) \leq \theta\left(P_{x}, P_{y}\right)^{2} \theta\left(P_{A}, P_{A}\right) \\
& \quad=\theta\left(P_{x}, P_{y}\right)^{2} \theta\left(P_{A}\right)^{2} .
\end{aligned}
$$

The square roots exist and are unique in $P^{*}\left(M_{n}(F)\right)$. Therefore $\theta\left(\left(P_{x}, P_{y}\right), P_{A}\right) \leq \theta\left(P_{x}, P_{y}\right) \theta\left(P_{A}\right)$.

Theorem 24 (polarisation identity). If $P_{A}, P_{B}$ are element of 2-FIPS $\left(M_{n}(F), \eta\right)$, then

$$
\begin{aligned}
4 \eta\left(P_{A}, P_{B}, s t\right)= & \theta\left(P_{A}+P_{B}\right)_{\alpha}^{2}-\theta\left(P_{A}-P_{B}\right)_{\alpha}^{2} \\
& +i \theta\left(P_{A}+i P_{B}\right)_{\alpha}^{2}-i \theta\left(P_{A}+i P_{B}\right)_{\alpha}^{2} .
\end{aligned}
$$

Proof. Consider

$$
\begin{aligned}
\theta & \left(P_{A}+P_{B}\right)_{\alpha}^{2}-\theta\left(P_{A}-P_{B}\right)_{\alpha}^{2}+i \theta\left(P_{A}+i P_{B}\right)_{\alpha}^{2} \\
& -i \theta\left(P_{A}+i P_{B}\right)_{\alpha}^{2}=\eta\left(P_{A}+P_{B}, P_{A}+P_{B},(s+t)^{2}\right) \\
& -\eta\left(P_{A}-P_{B}, P_{A}-P_{B},(s-t)^{2}\right) \\
& +i \eta\left(P_{A}+i P_{B}, P_{A}+i P_{B},(s+t)^{2}\right) \\
& -\eta\left(P_{A}-i P_{B}, P_{A}-i P_{B},(s-t)^{2}\right) \\
& =\eta\left(P_{A}+P_{B}, P_{A}+P_{B}, s^{2}+s t+s t+t^{2}\right) \\
& -\eta\left(P_{A}-P_{B}, P_{A}-P_{B}, s^{2}-s t-s t+t^{2}\right)
\end{aligned}
$$

$$
\begin{aligned}
& +i \eta\left(P_{A}+i P_{B}, P_{A}+i P_{B}, s^{2}+s t+s t+t^{2}\right) \\
& -i \eta\left(P_{A}-i P_{B}, P_{A}-i P_{B}, s^{2}-s t-s t+t^{2}\right) \\
& =\eta\left(P_{A}, P_{A}, s^{2}\right)+\eta\left(P_{A}, P_{B}, s t\right)+\eta\left(P_{B}, P_{A}, s t\right) \\
& +\eta\left(P_{B}, P_{B}, t^{2}\right)-\eta\left(P_{A}, P_{A}, s^{2}\right)-\eta\left(P_{A},-P_{B},-s t\right) \\
& -\eta\left(-P_{B}, P_{A},-s t\right)-\eta\left(-P_{B},-P_{B}, t^{2}\right) \\
& +i \eta\left(P_{A}, P_{A}, s^{2}\right)+i \eta\left(P_{A}, i P_{B}, s t\right)+i \eta\left(i P_{B}, P_{A}, s t\right) \\
& +i \eta\left(i P_{B}, i P_{B}, t^{2}\right)-i \eta\left(P_{A}, P_{A}, s^{2}\right) \\
& -i \eta\left(P_{A},-i P_{B},-s t\right)-i \eta\left(-i P_{B}, P_{A},-s t\right) \\
& -i \eta\left(-i P_{B},-i P_{B}, t^{2}\right)=\eta\left(P_{A}, P_{A}, s^{2}\right) \\
& +\eta\left(P_{A}, P_{B}, s t\right)+\eta\left(P_{B}, P_{A}, s t\right)+\eta\left(P_{B}, P_{B}, t^{2}\right) \\
& -\eta\left(P_{A}, P_{A}, s^{2}\right)-\eta\left(P_{A}, P_{B},-\frac{s t}{|-1|}\right) \\
& -\eta\left(P_{B}, P_{A},-\frac{s t}{|-1|}\right)-\eta\left(P_{B}, P_{B}, t^{2}\right) \\
& +i \eta\left(P_{A}, P_{A}, s^{2}\right)+i \eta\left(P_{A}, i P_{B}, s t\right)+i \eta\left(i P_{B}, P_{A}, s t\right) \\
& +i \eta\left(i P_{B}, i P_{B}, t^{2}\right)-i \eta\left(P_{A}, P_{A}, s^{2}\right) \\
& -i \eta\left(P_{A}, i P_{B},-\frac{s t}{|-1|}\right)-i \eta\left(i P_{B}, P_{A},-\frac{s t}{|-1|}\right) \\
& -i \eta\left(i P_{B}, i P_{B}, t^{2}\right)=\eta\left(P_{A}, P_{B}, s t\right)+\eta\left(P_{B}, P_{A}, s t\right) \\
& -\eta\left(P_{A}, P_{B},-s t\right)-\eta\left(P_{B}, P_{A},-s t\right)+i \eta\left(P_{A}, i P_{B}, s t\right) \\
& +i \eta\left(i P_{B}, P_{A}, s t\right)-i \eta\left(P_{A}, i P_{B},-s t\right) \\
& -i \eta\left(i P_{B}, P_{A},-s t\right)=\eta\left(P_{A}, P_{B}, s t\right)+\eta\left(P_{B}, P_{A}, s t\right) \\
& +\eta\left(P_{A}, P_{B}, s t\right)+\eta\left(P_{B}, P_{A}, s t\right) \\
& +i(-i) \eta\left(P_{A}, P_{B}, s t\right)+i(i) \eta\left(P_{B}, P_{A}, s t\right) \\
& +i(-i) \eta\left(P_{A}, P_{B}, s t\right)+i(i) \eta\left(P_{B}, P_{A}, s t\right) \\
& =\eta\left(P_{A}, P_{B}, s t\right)+\eta\left(P_{B}, P_{A}, s t\right)+\eta\left(P_{A}, P_{B}, s t\right) \\
& +\eta\left(P_{B}, P_{A}, s t\right)-\eta\left(P_{A}, P_{B}, s t\right)+\eta\left(P_{B}, P_{A}, s t\right) \\
& +\eta\left(P_{A}, P_{B}, s t\right)-\eta\left(P_{B}, P_{A}, s t\right)=4 \eta\left(P_{A}, P_{B}, s t\right) \text {. }
\end{aligned}
$$

Therefore,

$$
\begin{aligned}
4 \eta\left(P_{A}, P_{B}, s t\right)= & \theta\left(P_{A}+P_{B}\right)_{\alpha}^{2}-\theta\left(P_{A}-P_{B}\right)_{\alpha}^{2} \\
& +i \theta\left(P_{A}+i P_{B}\right)_{\alpha}^{2}-i \theta\left(P_{A}+i P_{B}\right)_{\alpha}^{2} .
\end{aligned}
$$




\section{Concluding Remarks}

In this paper the concept of inner product over fuzzy matrices has been discussed. We plan to extend our research work to (1) orthogonal of fuzzy matrices and (2) the Moore Penrose inverse and spectral inverse of fuzzy matrices.

\section{Conflict of Interests}

The authors declare that they do not have any conflict of interests regarding the publication of this paper.

\section{References}

[1] L. A. Zadeh, "Fuzzy sets," Information and Control, vol. 8, no. 3, pp. 338-353, 1965.

[2] R. Biswas, "Fuzzy inner product spaces and fuzzy norm functions," Information Sciences, vol. 53, no. 1-2, pp. 185-190, 1991.

[3] A. M. El-Abyad and H. M. El-Hamouly, "Fuzzy inner product spaces," Fuzzy Sets and Systems, vol. 44, no. 2, pp. 309-326, 1991.

[4] C. Felbin, "Finite-dimensional fuzzy normed linear space," Fuzzy Sets and Systems, vol. 48, no. 2, pp. 239-248, 1992.

[5] M. Z. Ragab and E. G. Emam, "The determinant and adjoint of a square fuzzy matrix," Information Sciences, vol. 84, no. 3-4, pp. 209-220, 1995.

[6] A. R. Meenakshi and R. Cokilavany, "On fuzzy 2-normed linear spaces," The Journal of Fuzzy Mathematics, vol. 9, no. 2, pp. 345351, 2001.

[7] T. Bag and S. K. Samanta, "Finite dimensional fuzzy normed linear spaces," Journal of Fuzzy Mathematics, vol. 11, no. 3, pp. 687-705, 2003.

[8] A. Nagoor Gani and G. Kalyani, "Binormed sequences in fuzzy matrices," Bulletin of Pure and Applied Science E, vol. 22, no. 2, pp. 445-451, 2003.

[9] A. N. Gani and G. Kalyani, "On fuzzy m-normed matrices," Bulletin of Pure and Applied Sciences E: Mathematics and Statistics, vol. 22, no. 1, pp. 1-11, 2003.

[10] P. Majumdar and S. K. Samanta, "On fuzzy inner product spaces," The Journal of Fuzzy Mathematics, vol. 16, no. 2, pp. 377392, 2008.

[11] A. Hasankhani, A. Nazari, and M. Saheli, "Some properties of fuzzy Hilbert spaces and norm of operators," Iranian Journal of Fuzzy Systems, vol. 7, no. 3, pp. 129-157, 2010.

[12] R. M. Somasundaram and T. Beaula, "Some aspects of 2-fuzzy 2-normed linear spaces," Bulletin of the Malaysian Mathematical Sciences Society, vol. 32, no. 2, pp. 211-221, 2009. 


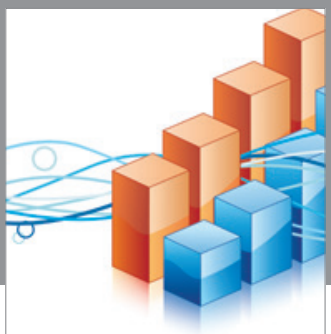

Advances in

Operations Research

vatem alat4

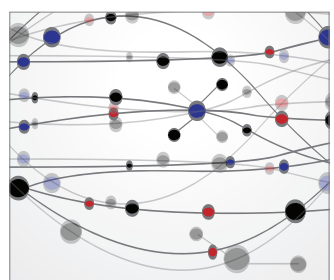

\section{The Scientific} World Journal
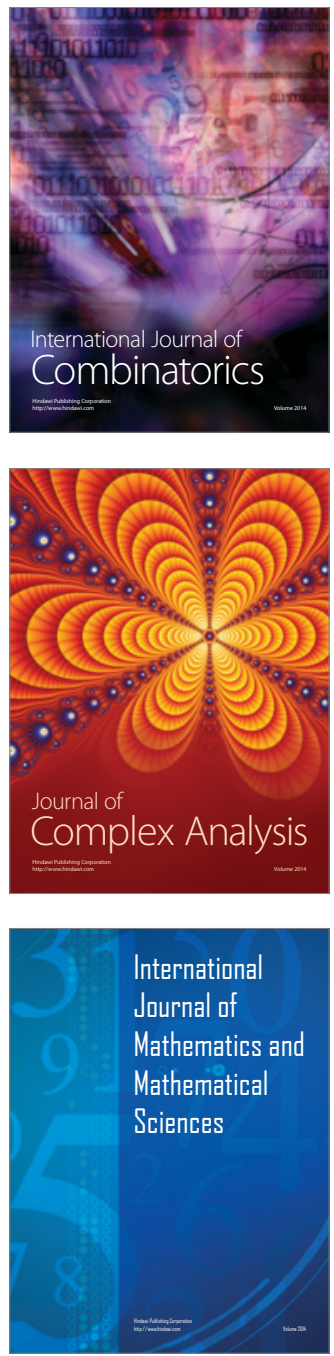
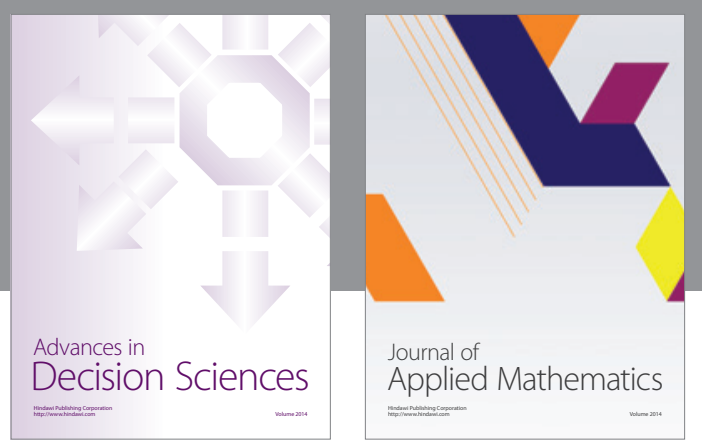

Algebra

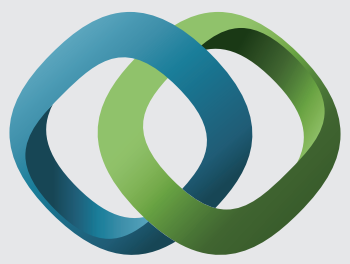

\section{Hindawi}

Submit your manuscripts at

http://www.hindawi.com
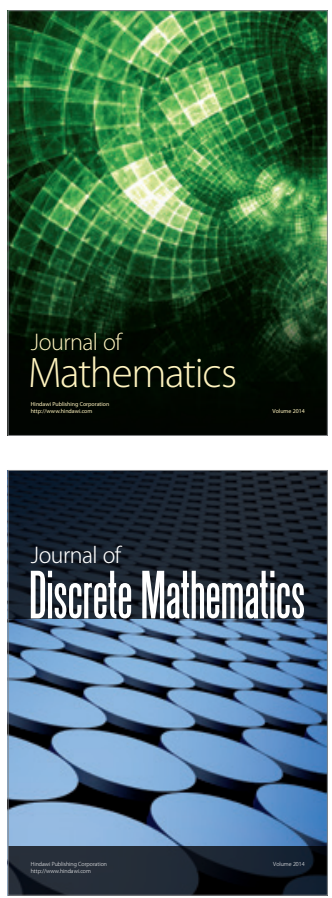

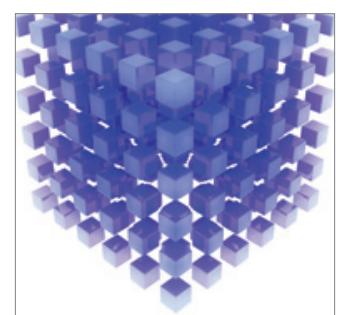

Mathematical Problems in Engineering
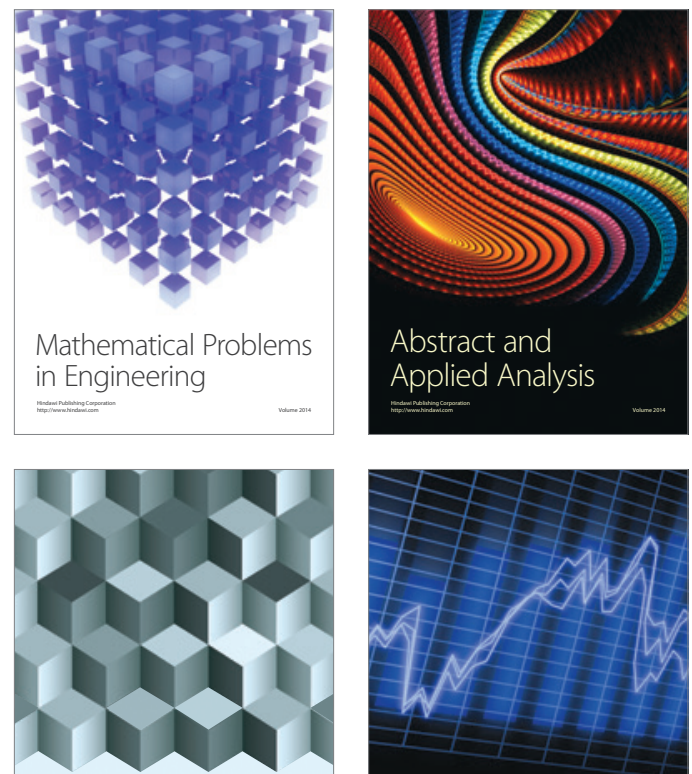

Journal of

Function Spaces

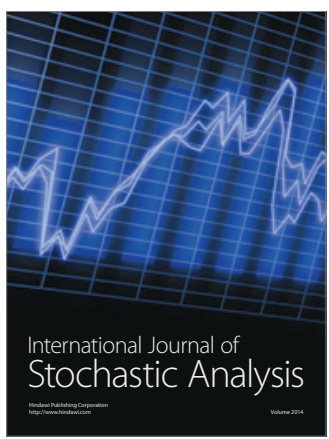

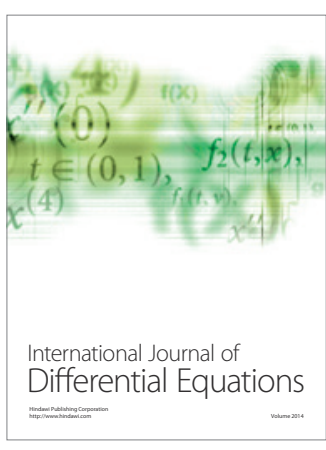
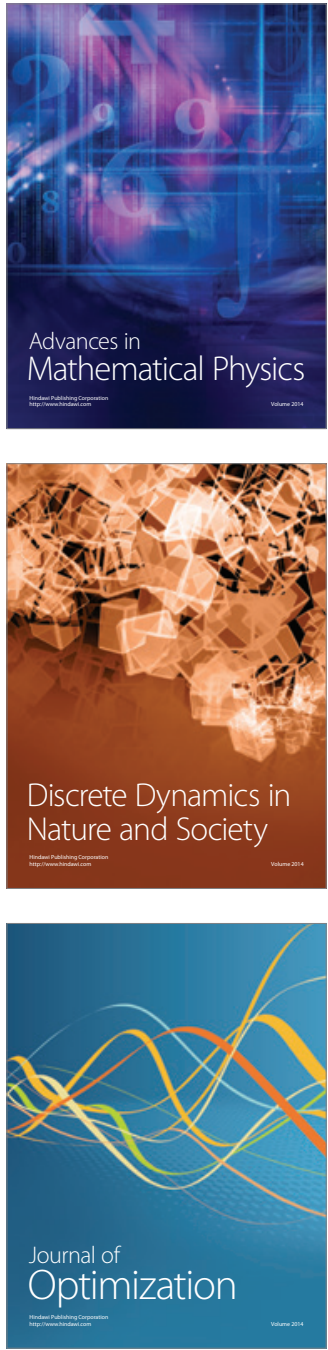